\title{
Pore Structure and Oxidation Stability of Double Walled Carbon Nanotube-derived Bucky Paper
}

H. Muramatsu, T. Hayashi, Y. A. Kim*, D. Shimamoto, Y. J. Kim, K. Tantrakarn and M. Endo Faculty of Engineering, Shinshu University 4-17-1 Wakasato, Nagano-shi 380-8553, Japan

M. Terrones

Advanced Materials Department, IPICYT, Camino a la Presa San José 2055, Col. Lomas 4a . Sección, San Luis Potosí 78216, México

\section{S. Dresselhaus}

Department of Physics, and Department of Electrical Engineering and Computer Science, Massachusetts Institute of Technology, Cambridge, MA 02139-4307, USA

\begin{abstract}
Thin, flexible and tough bucky paper fabricated by filtering a stable suspension of double walled carbon nanotubes (DWNTs) was characterized from the viewpoint of its pore structure and oxidation stability as compared with that derived from single-walled carbon nanotubes (SWNTs). The $\mathrm{N}_{2}$ adsorption isotherms of DWNTs and SWNTs at 77K were of type II and type IV, respectively, and also the micropore volume in DWNTs was three times greater than that in SWNTs. The onset temperature of oxidation in DWNTs was enhanced by ca. $200^{\circ} \mathrm{C}$ due to their coaxial structure. The expected high mechanical and electrical properties when combined with their high surface area and high structural integrity make the DWNTs bucky paper promising materials for numerous applications.

*Corresponding author: Tel: +81-26-269-5212, Fax: +81-26-269-5208, E-mail: yak@endomoribu.shinshu-u.ac.jp
\end{abstract}

\section{Introduction}

In the last decade, much attention has been paid to the synthesis of double walled carbon nanotubes (DWNTs) [1-7], not only from the point of basic science but also from an engineering study point, due to their unique hybrid structure between single walled carbon nanotubes (SWNT) and multi-walled carbon nanotubes (MWNT). Furthermore, DWNTs have small diameter (below 2nm) which is very similar to SWNTs, and their corresponding structural 
stability is considerably higher than that of SWNTs [8], due to their coaxial structure. Thus, by exploiting their thin diameter (below 2nm) and their structural stability, DWNTs are expected to be utilized in a wide range of applications $[9,10]$.

Recently we fabricated highly purified DWNT web [1] through the right combination of a catalytic chemical vapor deposition (CCVD) method [11, 12] with an optimized two-step purification process. Careful high resolution transmission electron microscopy (HRTEM) observations confirmed a high yield of DWNTs (95\%) in bundles, and magnetic susceptibility studies confirmed the high purity of DWNTs through their diamagnetic behavior [13]. On the other hand, SWNT bucky paper, fabricated by a filtering procedure from highly stable suspensions of SWNTs has exhibited possible uses as hydrogen storage material, anode materials in lithium ion batteries, and actuators or artificial muscle and sensors [14-18]. This is due to an intrinsic high accessible surface area (derived from their nano-sized diameter), and high handleability. In this sense, it is very important to evaluate the surface properties of DWNT bucky paper, including its oxidation stability as compared with that of SWNTs, especially in order to understand the structural differences between bundles of single graphene tubules and DWNTs ropes.

Here, we carried out a comparative study of the pore structure and the oxidation stability between DWNT- and SWNT-derived bucky papers. It is expected that our study will spur further theoretical and experimental study of the basic science and technology of DWNTs, thus eventually replacing SWNTs in various applications fields.

\section{Experimental}

The synthesis of DWNTs was carried out by a catalytic chemical vapor deposition (CCVD) method utilizing a conditioning catalyst $\left(\mathrm{Mo} / \mathrm{Al}_{2} \mathrm{O}_{3}\right)$ at one end of the furnace, and the nanotube catalyst $(\mathrm{Fe} / \mathrm{MgO})$ in the middle part of the furnace [1]. Subsequently, a $\mathrm{CH}_{4}+\mathrm{Ar}$ (1:1) mixture was fed into the reactor for typically 10 minutes at $875{ }^{\circ} \mathrm{C}$. In order to obtain highly pure DWNT-web, a two-step purification process was applied to the synthesized products. In particular, a hydrochloric acid $\left(18 \%, 100{ }^{\circ} \mathrm{C}, 10 \mathrm{hrs}\right)$ treatment was carried out in order to remove the $\mathrm{MgO}$ and iron catalyst, followed by air oxidation at $500{ }^{\circ} \mathrm{C}$ for 30 minutes. Then, we prepared a highly dispersed solution containing DWNTs (15mg in 100ml ethanol) using ultra-sonication (KUBOTA UP50H) for 30 minutes, without any surfactant. By filtering a stable suspension of DWNTs (polytetrafluoroethylene (PTFE) filter, $1 \mu \mathrm{m}$ ), drying it for 24hrs in vacuum and peeling off the resulting bucky paper from the PTFE filter very carefully, we finally 
obtained a black DWNT bucky paper which is thin, flexible and tough enough mechanically to fold in origami. Highly purified SWNTs (bucky pearls) purchased from Carbon Nano-technology Inc. were used without further purification.

HRTEM (JEOL2010FEF), field emission scanning microscopy (FE-SEM) (JSM6335Fs), Raman spectroscopy (Kaiser HoloLab5000 system: 532nm Ar-ion laser line) and X-ray diffraction (Rigaku RINT-2200, $\mathrm{CuK}_{\alpha}$ ) characterizations were carried out in order to obtain structural information. We also carried out nitrogen adsorption measurements at $77 \mathrm{~K}$ (ASAP2010) after pretreatment at 553K for $6 \mathrm{~h}$ and thermogravitic analysis (Shimadzu DTG-60/60, 1\% $\mathrm{O}_{2}+$ Argon gas) on DWNT- and SWNT-bucky papers.

\section{Results and discussion}

It is well known that nano-sized carbon nanotubes have a high tendency toward self-aggregation due to strong Van der Waals forces [19]. By utilizing this intrinsic nature of carbon nanotubes, we are able to fabricate paper-like sheets (so called bucky paper) from carbon nanotubes dispersed in solution. At first, we prepared a stable suspension of DWNTs with the help of ultra-sonication (30 minutes). Here, it is noteworthy that no surfactant (e.g., Triton X-100) was used. This is very important for fabricating high purity bucky paper. Then, by pouring a stable suspension of nanotubes into a PTFE filter-attached funnel very carefully, very thin (ca. $30 \mu \mathrm{m}$ ), round (diameter $=3.4 \mathrm{~cm}$ ), light (weight $=$ ca. $15 \mathrm{mg}$ ) and black bucky paper was produced (see Fig. 1 (a) and (b)). As expected, bucky paper is the result from the physical entanglement of DWNT bundles (10-30nm) (Fig. 1 (c)). From the cross-sectional HRTEM image (Fig. 1 (d)), we observed that our tubes consist of relatively round, small and homogeneous-sized (below $2 \mathrm{~nm}$ in the outer shell) two concentric individual tubules. Furthermore, these co-axial tubes are packed in a hexagonal array. For SWNT-derived bucky paper, physical fracture occurred easily when peeling the bucky paper off from the PTFE filter, even though we applied exactly the same procedure and also used the same amount of tubes. This difference in structural integrity between DWNT- and SWNT-derived bucky papers is thought to be derived mainly from the different lengths of tubes, because the longer the tubes, the stronger is the mechanical robustness of the intermingled bundle. In this sense, the mechanical properties of DWNT-bucky paper have to be evaluated in the near-future in terms of tube length and bundle size. On top of that, SWNTs exhibit a wide diameter distribution (Fig. 1 (f)) and bundle sizes (Fig. 1 (e)). The great difficulty in observing cross-sectional images (when the nanotube bundle is bent or curled, and passes through the electron beam) for the case of SWNTs indirectly indicates a short tube length, which could be derived from the intrinsic characteristics of the Hipco process [20]. 
In order to confirm the diameter distribution and also the structural perfection of the materials, Raman and X-ray diffraction studies were carried out on DWNT- and SWNT-derived bucky papers, respectively. Figure 2 shows Raman spectra in the low frequency $(\mathrm{RBM}=$ radial breathing mode), and high frequency (tangential G-band mode) ranges, respectively. It is noteworthy that the D-band (defect-induced mode) for DWNTs is negligible in the Raman spectra. We also noticed relatively well-developed (100) and (110) diffraction lines from XRD measurements in our DWNTs, thus indicating that our tubes are highly crystalline and contain a low concentration of structural defects. It is well known that the RBM frequency is inversely related to the tube diameters [21]. For DWNTs, Raman RBM features above $250 \mathrm{~cm}^{-1}$ correspond to the inner tubes, whereas peaks below $250 \mathrm{~cm}^{-1}$ are associated with the outer tubular shells. The values in Fig. 2 indicate the calculated diameters using the following equation $\omega_{R B M}=218.3 / d_{t}+15.9$, where $d_{t}$ is the tube diameter $(n m)$ and $\omega_{R B M}$, is the RBM frequency $\left(\mathrm{cm}^{-1}\right)$ [21]. Therefore, the RBM spectra of our DWNTs indicate the presence of two pairs of tube diameters: $(0.74 \mathrm{~nm} ; 1.41 \mathrm{~nm})$ and $(0.87 \mathrm{~nm} ; 1.56 \mathrm{~nm})$, which is consistent with HRTEM observations (Fig. 1 (d)). On the other hand, RBM region for SWNTs indicate a wide distribution of diameters: $(1.26 \mathrm{~nm}),(1.01 \mathrm{~nm})$ and $(0.85 \mathrm{~nm})$. In our DWNTs, the rather sharp $\mathrm{G}^{+}$ peak at $1592 \mathrm{~cm}^{-1}$, the $\mathrm{G}^{-}$shoulder peak at $1570 \mathrm{~cm}^{-1}$ (corresponding to semiconducting tubes), and the low intensity of the asymmetric Raman features located at ca. $1550 \mathrm{~cm}^{-1}$ (corresponding to metallic tubes) [22] are indicative that suggests a larger concentration of semi-conducting tubes.

The pore structure and surface properties of SWNT- and DWNT-derived bucky papers were studied comparatively using the $\mathrm{N}_{2}$ adsorption isotherms at $77 \mathrm{~K}$ (Figure 3 (a)). Both samples basically followed the type IV isotherms with hysteresis. However, DWNTs exhibited more steady uptake of $\mathrm{N}_{2}$ at a medium relative pressure $\left(P / P_{0}\right)$ and abrupt uptake of $\mathrm{N}_{2}$ at the high relative pressure (above 0.9), compared with that of SWNT which shows a hysteresis loop at a lower relative pressure around 0.5 [23]. An abrupt increase of $\mathrm{N}_{2}$ uptake below $\mathrm{P} / \mathrm{P}_{0}=0.1$ (see inset of Figure 3 (a)) strongly suggests the presence of micropores smaller than $-1.5 \mathrm{~nm}$. Furthermore, below $P / P_{0}<0.02$, the higher uptake of $N_{2}$ in DWNTs when compared to SWNTs is directly related to the three times large micropore volume observed in DWNTs, although the total surface area of DWNTs $\left(569 \mathrm{~m}^{2} / \mathrm{g}\right)$ is lower than that of SWNTs $\left(642 \mathrm{~m}^{2} / \mathrm{g}\right)$. Besides, a sharper angle of DWNT between the steep initial rise below 0.01 and the plateau implies the more uniform pore structure than those of SWNT. In order to elucidate the micropore structure of the samples, the adsorption data in an extremely low region of relative pressure $\mathrm{P} / \mathrm{P}_{0}$ should be considered in detail. Figure 3 (b) presents the details of the adsorption behavior in the low 
relative pressure, clearly showing that the beginning point of $\mathrm{N}_{2}$ adsorption is in the vicinity of $\mathrm{P} / \mathrm{P}_{0}=10^{-6}$. We summarize the pore structure of DWNT- and SWNT-derived bucky papers comparatively in Table 1 . The pore size distributions for both samples were obtained from $\mathrm{N}_{2}$ adsorption isotherms using the density functional theory (DFT) method [24], as shown in Fig. 3 (c). From this figure, both samples exhibit similar micro- and mesopore distributions. But, the clearly observed small micropores (ca. $0.8 \mathrm{~nm}$ ) and the three times large micropore volume (see Table 1) in DWNTs are ascribed to well-developed intertube-cavities due to the high hexagonal stacking order, which is confirmed by XRD studies [13]. In addition, a large portion of pores ranging from 20 to $100 \mathrm{~nm}$ in DWNTs (Fig. 3 (b)) is thought to be caused by empty spaces created by highly intermingled long bundles.

Finally1, a comparative study of the resistance to oxidation for both bucky papers was carried out using a thermal gravimetric analysis in an argon and oxygen (1\%) gas mixture (Fig. 4). By taking the first derivative of Fig. 4, we can obtain the temperature at which the rate of combustion is highest. The results demonstrate that DWNTs oxidized at a significantly higher temperature (ca. $717^{\circ} \mathrm{C}$ ) when compared to SWNTs (ca. $541{ }^{\circ} \mathrm{C}$ ). We believe that the high resistance to oxidation of DWNTs is derived from the structural features (namely the "coaxial structure”) as well as the high crystallinity of the bundles. We could easily discard the possibility of iron- catalyst particles and amorphous carbon in our samples because both samples (SWNTs and DWNTs) were highly purified by oxidation and hydrochloric acid processes. It is very interesting to note that DWNTs are also structurally stable when they are thermally treated up to $2000^{\circ} \mathrm{C}$ [8]. Therefore, it will be very important now to compare the structural and oxidation stability of nanotubes as a function of the number of concentric walls within multi-walled carbon nanotubes (MWNTs).

\section{Conclusion}

In this work, we carried out comparative $\mathrm{N}_{2}$ adsorption studies at $77 \mathrm{~K}$ and thermogravitic analysis on SWNT- and DWNT-derived bucky papers. We noted that the easy fabrication of thin, flexible and tough DWNT-derived bucky paper by filtering the stable suspension of DWNTs (especially without using surfactant), is due to the presence of long and well-bundled DWNTs whereby van der Waals forces play an important role of the structural integrity of bucky papers. The low surface area and the large micropore volumes observed for DWNTs results from the purity of the material (e.g., low concentration of amorphous carbon and also metal particles) and the high crystallinity (tubes are hexagonally packed, maybe due to the high elastic modulus of 
individual DWNTs). These values were superior to those observed in SWNTs. It is clear that the co-axial structure of DWNTs directly contributes to an enhanced oxidation resistance of ca. $200^{\circ} \mathrm{C}$. We envisage that the high structural stability and electrical conductivity of DWNTs, combined with their relatively high surface area and high oxidation resistance indicate that DWNT-derived bucky paper will certainly find uses in various fields (e.g., actuators, composites, field emission devices, sensors, etc).

\section{Acknowledgements}

This work was supported by the CLUSTER of Ministry of Education, Culture, Sports, Science and Technology. M.S.D acknowledges support from US-NSF grant DMR 04-05538. M.T. thanks CONACYT-México for grants: 37589-U, 41464-Inter American Collaboration, PUE-2004-CO2-9 Fondo Mixto de Puebla and 2004-01-013/SALUD CONACYT. M.S.D. and M.T. also thank MIT-CONACYT collaboration program.

\section{Figure captions}

Fig.1 (a) Photograph of round (diameter $=3.4 \mathrm{~cm}$ ) and black DWNT-derived bucky paper, (b) low-resolution FE-SEM image shows highly thin (ca, 30 $\mu \mathrm{m}$ ) paper obtained from 20mg DWNTs, (c) FE-SEM and low resolution TEM (see, inset) images exhibit intermingled bundles of DWNTs, (d) cross-sectional HR-TEM image clearly show that our tubes consist of small sized, round and homogeneous two concentric shells. (e) FE-SEM and low resolution TEM (see, inset) images of highly purified SWNTs, showing a wide distribution of bundle sizes (f) cross-sectional HR-TEM image of SWNTs shows wide diameter distribution.

Fig. 2 Raman spectra of SWNT- and DWNT-derived bucky papers, respectively, using a $532 \mathrm{~nm}$ laser line. Note that there is no appearance of a D band feature from the Raman spectra of DWNTs, due to low concentration of defects.

Fig. 3 Adsorption isotherm of SWNT- and DWNT-derived bucky papers (inset shows a comparative plot of the adsorption on both samples below $P / P_{0}=0.1$ ). The abscissa is expressed by linear (a) and the logarithm (b) of the relative pressure $\left(\mathrm{P} / \mathrm{P}_{0}\right)$. (c) micropore size distribution of SWNT- and DWNT-derived bucky papers using the DFT method. The solid line indicates DWNTs whereas the dotted line indicates SWNTs. Note that small micropores below $0.8 \mathrm{~nm}$ corresponding to interstitial pores were observed in DWNTs. 
Fig. 4 Thermogravitic analysis of SWNT- and DWNT-derived bucky papers in an argon and oxygen (1\%) gas mixture. It is noteworthy that an improved oxidation stability in DWNTs by ca. $200^{\circ} \mathrm{C}$ is derived from their coaxial structure.

Table 1 Pore analysis of SWNT- and DWNT-derived bucky papers

\begin{tabular}{lcccc}
\hline Samples & $\mathrm{S}_{\mathrm{BET}}\left(\mathrm{m}_{2} / \mathrm{g}\right)$ & $\mathrm{S}_{\text {micropore }}\left(\mathrm{m}^{2} / \mathrm{g}\right)$ & $\mathrm{V}_{\text {tot }}\left(\mathrm{m}^{3} / \mathrm{g}\right)$ & $\mathrm{V}_{\text {micropore }}\left(\mathrm{m}^{3} / \mathrm{g}\right)$ \\
\hline $\begin{array}{l}\text { DWNT-derived } \\
\text { bucky paper }\end{array}$ & 569 & 150 & 2.05 & 0.11 \\
\hline $\begin{array}{l}\text { SWNT-derived } \\
\text { bucky paper }\end{array}$ & 642 & 11 & 1.17 & 0.04 \\
\hline
\end{tabular}

\section{References}

[1] M. Endo, H. Muramatsu, T. Hayashi, Y. A. Kim, M. Terrones, M. S. Dresselhaus, Nature 433 (2005) 476.

[2] S. Bandow, M. Takizawa, K. Hirahara, M. Yudasaka, S. Iijima, Chem. Phys. Lett. 337 (2001) 48.

[3] J. Wei, L. Ci, B. Jiang, Y. Li, X. Zhang, H. Zhu, C. Xu, D. Wu, J. Mater. Chem. 13 (2003) 1340.

[4] S. C. Lyu, B. C. Liu, C. J. Lee, Chem. Mater. 15 (2003) 3951.

[5] T. Sugai, H. Yoshida, T. Shimada, T. Okazaki, H. Shinohara, Nano Lett. 3 (2003) 769.

[6] T. Hiraoka, T. Kawakubo, J. Kimura, R. Taniguchi, A. Okamoto, T. Okazaki, T. Sugai, Y. Ozeki, M. Yoshikawa, H. Shinohara, Chem. Phys. Lett. 382 (2003) 679.

[7] E. Flahaut, R. Basca, A. Peigney, C. Laurent, Chem. Commun., (2003) 1442.

[8] Y. A. Kim, H. Muramatsu, T. Hayashi, M. Endo, M. Terrones and M. S. Dresselhaus, Chem. Phys. Lett., 398 (2004) 87.

[9] H. Kurachi, S. Uemura, J. Yotani, T. Nagasako, H. Yamada, T. Ezaki, t. Maesoba, R. Loutfy, A. Moravsky, T. Nakagawa, S. Katagiri, Y. Saito, Proceedings of 21st International Display Research Conference/8th International Display Workshops, (2001) 1245.

[10] R.H. Baughman, A.A. Zakhidov, W.A. de Heer, Science 297 (2002) 787.

[11] A. Oberlin, M. Endo and T. Koyama, J. Crys. Growth, 32 (1976) 335.

[12] M. Endo, CHEMTECH, (1988) 568.

[13] M. Endo et al., (unpublished) 
[14] C. Liu, Y.Y. Fan, M. Liu, H. T. Cong, H.M. Cheng, M.S. Dresselhaus, Science 286 (1999) 1127.

[15] A.S. Claye, J.E. Fischer, C.B. Huffman, A.G. Rinzler, R.E. Smalley, J. Electrochem. Soc. 147 (8), (2000) 2845.

[16] G.U. Sumanasekera, C.K.W. Adu, S. Fang, P. C. Eklund, Phys. Rev. Lett. 85 (2000) 1096.

[17] R.H. Baughman, C. Cui, A.A. Zakhidov, Z. lqbal, J.N. Barisci, G.M. Spinks, G.G. Wallace, A. Mazzoldi, D. D. Rossi, A.G. Rinzler, O. Jaschinski, S. Roth, M. Kertesz, Science, 284 (1999) 1340.

[18] U. Vohrer, I. Kolaric, M.H. Haque, S. Roth, U. Detlaff-Weglikowska, Carbon 42 (2004) 1159.

[19] A. Thess, R. Lee, P. Nikolaev, H. Dai, P. Petit, J. Robert, C. Xu, Y.-H. Lee, S.-G. Kim, A.G. Rinzler, D. T. Colbert, G. E. Scuseria, D. Tomanek, J. E. Fischer, R. E. Smalley, Science, 273 (1996) 483.

[20] M.J. Bronikowski, P.A. Willis, D.T. Colbert, K. A. Smith, R.E. Smalley, Journal of Vacuum Science \& Technology A-Vacuum Surfaces \& Films, 19 (2001) 1800.

[21] A. Jorio, M.A. Pimenta, A. G. Souza Filho, R. Saito, G. Dresselhaus and M. S. Dresselhaus, New. J. Phys. 5 (2003) 139.

[22] P. Corio, M.L.A. Temperini, P.S. Santos, J.V. Romero, J.G. Huber, C.A. Luengo, S.D.M. Brown, M.S. Dresselhaus, G. Dresselhaus, M.S.S. Dantas, C.F. Liete, F. Matinaga, J.C. Gonzalez, M.A. Pimneta, Chem. Phys. Lett. 350 (2001) 373.

[23] C.M. Yang, K. Kaneko, M. Yudasaka, S. Iijima, Nano Lett., 2 (2002) 385.

[24] P. Kluson, S.J. Scaife, Chem. Biochem. Eng. Q. 15 (2001) 117. 